\title{
A Broadband Vibration-Based Energy Harvester Using an Array of Piezoelectric Beams Connected by Springs
}

\author{
V. Meruane and K. Pichara \\ Department of Mechanical Engineering, Universidad de Chile, Beauchef 851, 9370456 Santiago, Chile \\ Correspondence should be addressed to V. Meruane; vmeruane@ing.uchile.cl
}

Received 2 June 2015; Revised 25 August 2015; Accepted 27 August 2015

Academic Editor: Carlo Trigona

Copyright ( 2016 V. Meruane and K. Pichara. This is an open access article distributed under the Creative Commons Attribution License, which permits unrestricted use, distribution, and reproduction in any medium, provided the original work is properly cited.

Piezoelectric cantilevered beams have been widely used as vibration-based energy harvesters. Nevertheless, these devices have a narrow frequency band and if the excitation is slightly different there is a significant drop in the level of power generated. To handle this problem, the present investigation proposes the use of an array of piezoelectric cantilevered beams connected by springs as a broadband vibration-based energy harvester. The equations for the voltage and power output of the system are derived based on the analytical solution of the piezoelectric cantilevered energy harvester with Euler-Bernoulli beam assumptions. To study the advantages and disadvantages of the proposed system, the results are compared with those of an array of disconnected beams (with no springs). The analytical model is validated with experimental measurements of three bimorph beams with and without springs. The results show that connecting the array of beams with springs allows increasing the frequency band of operation and increasing the amount of power generated.

\section{Introduction}

The idea of harvesting energy from the environment resulting in self-powered monitoring systems is fascinating and has attracted the attention of many researchers. This is demonstrated by the large numbers of investigations in the area [1-8]. Examples of remote sensors that could benefit from energy harvesting are weather monitoring, sea surface water monitoring, tactical military surveillance, monitoring of equipment and structures, oceanography, and volcano and seismic monitoring to name a few. For most of these applications, renewable power sources are needed for longterm remote monitoring. Some of the different power sources available are thermoelectric energy, mechanical vibrations, hydroelectric power, wind energy, solar power, and ambient radio frequency.

Mechanical vibrations are an attractive source due to their high availability in certain environments. The transduction mechanisms that can be used to convert ambient vibration into electrical energy are electromagnetic, electrostatic, and piezoelectric. Among these mechanisms, piezoelectric transduction has received great attention in the last years due to its ability to directly convert strain energy into usable electric energy $[1,9]$. In piezoelectric energy harvesting the mechanical energy of vibrations is transformed by a piezoelectric material to electrical energy. When a piezoelectric material is deformed, the central molecules in the crystal become polarized and form a dipole. If the dipoles are arranged, then two surfaces of the material become positively and negatively charged. This property can be exploited to transform mechanical strain into electrical energy. Figure 1 presents a scheme of two cantilever piezoelectric power generators. The generators are submitted to base excitation, which causes the beams to vibrate, inducing an alternating voltage. The voltage generated by the piezoelectric elements can be used to power a device directly or to charge a rechargeable battery. In the case of a unimorph beam, a layer of piezoceramic material is attached to a metal layer (substructure), whereas in a bimorph configuration a metal layer is sandwiched between two piezoelectric layers. In both cases, a proof mass is attached at the end to increase the strain.

The main problem of conventional energy harvesters is their narrow frequency band of operation. Conventional energy harvesters, as the ones shown in Figure 1, have 


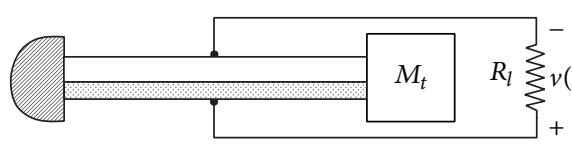

$\square$ Piezoceramic

Substructure

(a)

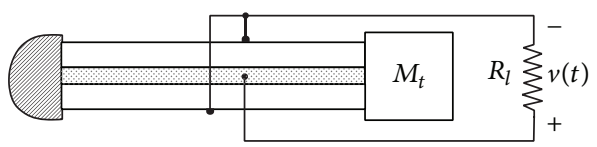

$\square$ Piezoceramic

Substructure

(b)

FIGURE 1: Cantilever piezoelectric energy configurations: (a) unimorph, (b) bimorph (parallel connection).

an operating frequency equal to their resonance frequency, and if the excitation is slightly different there is a significant drop in the level of power generated. This problem has led to the search for broadband energy harvesters. Twiefel and Westermann [10] present a survey of broadband techniques for vibration-based energy harvesting. These techniques are classified as follows:

(i) Linear generators: arrays, combined modes.

(ii) Nonlinear generators: bi/multiple stable, vibroimpact, resonant tuning.

(iii) Advanced electronic networks: switching networks, synthetic impedance.

According to the researchers there is no universal solution; depending on the application, one technique may be better than the others. For example, linear generators are more efficient for stochastic excitations whereas nonlinear techniques are more suitable for harmonic excitations or very low excitation frequencies.

In the case of linear generators, recent research has been focused on developing systems that exhibit multiple resonances that are closely spaced. The simplest solution is to use multiple cantilevers. The cantilevers are built with different geometries (large, tip mass or width) so each beam has a slightly different resonant frequency, thus increasing the bandwidth of the full system. A first design of a cantilever array was presented by Shahruz $[11,12]$. He concluded that if the dimensions of the beams and masses are chosen appropriately, then the system performs as a broadband harvester. However, the maximal frequency band is limited and is independent of the beams dimensions. This approach has been studied extensively since then [13-15].

Another method is to use structures with two or more degrees of freedom, resulting in two or more resonant frequencies that are closely spaced [16-19]. Z. Yang and J. Yang [18] analyzed the power generated by the flexural vibrations of two piezoelectric beams connected by springs; they showed that this structure can be used as a broadband energy harvester. Nevertheless, the model presented by the authors is restricted to two beams and the extension to more than two beams is not trivial. Springs have also been used in MEMS-based energy harvesters, where it has been demonstrated that the use of nonlinear springs increases the generated power [20-22].

The research community related to piezoelectric energy harvesting is very wide and includes researchers from mechanical, electrical, materials, and civil engineering. This has led to the development of many different models for the same problem: a piezoelectric cantilevered beam. Most of these models use simplifications, such as oversimplifying of the piezoelectric coupling as viscous damping [23], using of the static deflection of the beam in a dynamic model $[24,25]$, no inclusion of the piezoelectric effect in the mechanical model [26], or approximate solutions as the Rayleigh-Ritz approximation [27, 28]. Erturk and Inman [29] provide a comprehensive discussion of the different simplifications found in literature to model piezoelectric beams, highlighting the common indiscretions and providing corrections and necessary clarifications for researchers from different engineering fields. The best solution can be obtained by solving the continuous electromechanical model analytically. Different approaches for solving the continuous model can be found in literature [30-33].

In this paper we present a general approach to characterize multiple piezoelectric beams connected with springs. The analytical solution of the cantilevered piezoelectric energy harvester with Euler-Bernoulli beam assumptions proposed by Erturk and Inman [33] is extended to the case of an array of piezoelectric beams connected by springs. The proposed configuration is attractive since it combines the properties of beam arrays and of systems with multiple degrees of freedom. Furthermore, the results demonstrate that connecting an array of beams with springs allows increasing the frequency band of operation and increasing the amount of power generated when compared with an array of disconnected beams.

The remainder of this work is organized as follows. Section 2 presents the structure under investigation and the methodology to compute its modes shapes and natural frequencies. Section 3 provides the derivation of the electromechanical equations of a bimorph cantilever beam. In Section 4 the equations of the output voltage and power of an array of bimorph cantilever beams connected by springs are derived. Section 5 presents analytical and experimental results for different combinations of tip masses and springs constants, comparing the results with those of an array of disconnected beams (with no springs). Finally, conclusions and forthcoming work are presented in Section 6. 


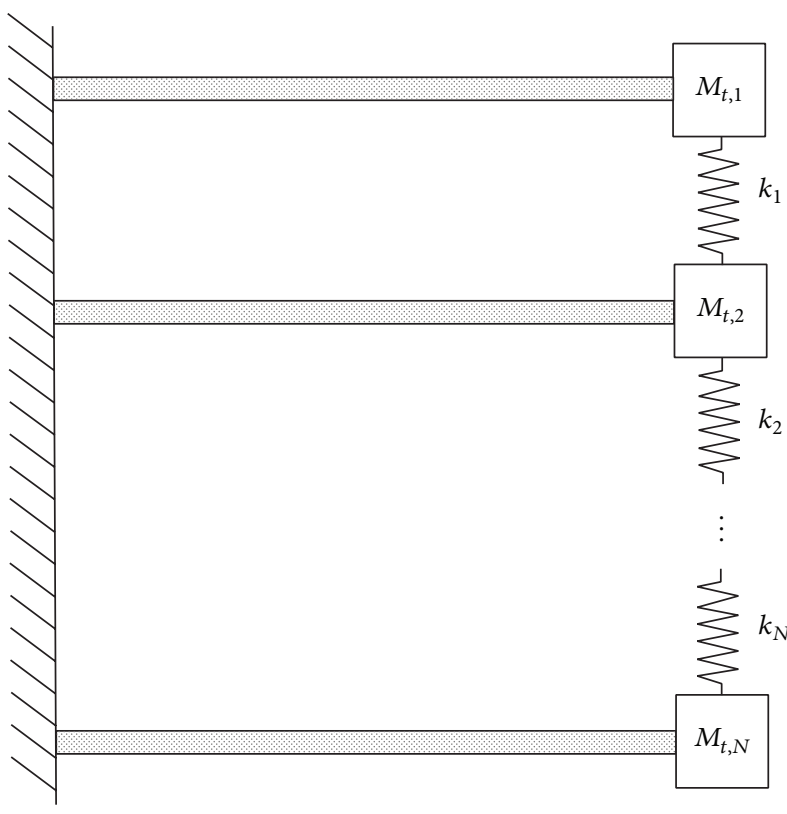

(a) Full system

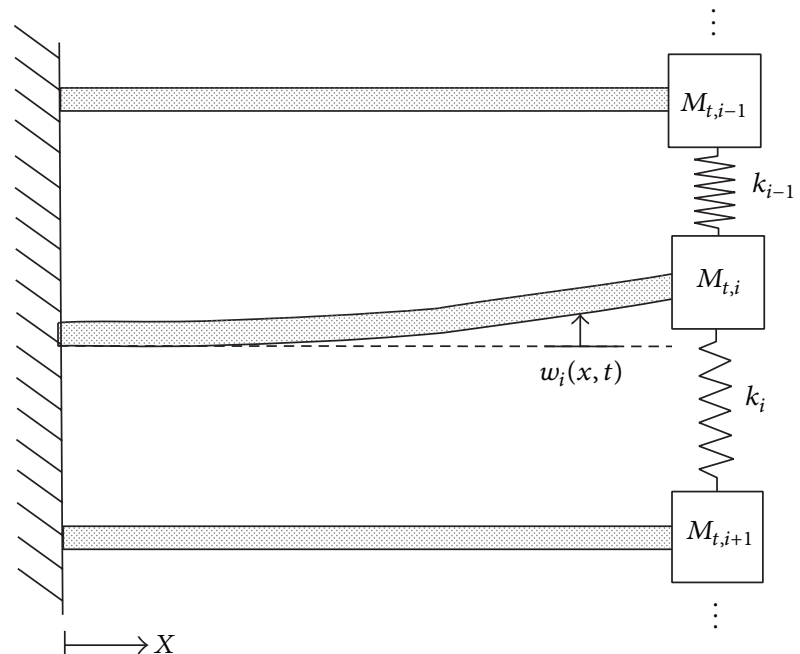

(b) Scheme of the $i$ th beam

FIGURE 2: Structure consisting in an array of piezoelectric beams connected by springs.

\section{Structure and Vibration Modes}

The structure under investigation is an array of piezoelectric beams connected by springs, as illustrated in Figure 2(a). All beams are clamped at the left-end to a vertical wall. The rightend of each beam is connected to a concentrated tip mass and to its neighbors beams by springs. The inertia of the concentrated masses is neglected. All beams have identical dimensions and the only differences are the tip masses.

To derive the vibration modes of this system, it is necessary to study the boundary conditions of each beam separately. Looking at the ith beam, as shown in Figure 2(b), and assuming a separation-of-variables solution, the transverse displacement of the $i$ th beam can be expressed as $w_{i}(x, t)=$ $\phi_{i}(x) \eta(t)$. The function $\phi_{i}(x)$ represents the mode shape and depends on the boundary conditions. In an Euler-Bernoulli beam, the general solution for $\phi_{i}(x)$ is

$$
\begin{aligned}
\phi_{i}(x)= & \lambda_{1}^{i} \sin (\beta x)+\lambda_{2}^{i} \cos (\beta x)+\lambda_{3}^{i} \sinh (\beta x) \\
& +\lambda_{4}^{i} \cosh (\beta x) .
\end{aligned}
$$

The boundary conditions at the left-end are

$$
\begin{aligned}
\phi_{i}(0) & =0, \\
\frac{\partial \phi_{i}(0)}{\partial x} & =0,
\end{aligned}
$$

which implies that

$$
\begin{aligned}
& \lambda_{4}^{i}=-\lambda_{2}^{i}, \\
& \lambda_{3}^{i}=-\lambda_{1}^{i} .
\end{aligned}
$$

Therefore the mode shape can be written as

$$
\begin{aligned}
\phi_{i}(x)= & \lambda_{1}^{i}[\sin (\beta x)-\sinh (\beta x)] \\
& +\lambda_{2}^{i}[\cos (\beta x)-\cosh (\beta x)] .
\end{aligned}
$$

At the right-end, the bending moment and shear force must satisfy the following conditions:

$$
\begin{gathered}
Y I \frac{\partial^{2} w_{i}}{\partial x^{2}}=0 \\
\frac{\partial}{\partial x}\left[Y I \frac{\partial^{2} w_{i}}{\partial x^{2}}\right]= \\
-M_{t, i} \frac{\partial^{2} w_{i}}{\partial t^{2}}-k_{i}\left(w_{i}-w_{i+1}\right) \\
-k_{i-1}\left(w_{i}-w_{i-1}\right)
\end{gathered}
$$

where $Y$ is Young's modulus, $I$ is the area moment of inertia of the cross section, $M_{t, i}$ is the $i$ th tip mass, and $k_{i}$ is the stiffness of the $i$ th spring. Replacing that $w_{i}(x, t)=\phi_{i}(x) \eta(t)$ and $\partial^{2} \eta(t) / \partial t^{2}=-\omega_{n}^{2} \eta(t), \omega_{n}$ being the natural frequency, yields

$$
\begin{aligned}
\frac{\partial^{2} \phi_{i}(L)}{\partial x^{2}}= & 0, \\
Y I \frac{\partial^{3} \phi_{i}(L)}{\partial x^{3}}= & \omega_{n}^{2} M_{t, i} \phi_{i}(L)-k_{i}\left[\phi_{i}(L)-\phi_{i+1}(L)\right] \\
& -k_{i-1}\left[\phi_{i}(L)-\phi_{i-1}(L)\right],
\end{aligned}
$$


where $L$ is the length of the beam. Replacing the definition of $\phi_{i}(x)$ from (4), the following equations are obtained:

$$
\left[\begin{array}{cccccc}
0 & 0 & \alpha_{1} & \alpha_{2} & 0 & 0 \\
\alpha_{3}\left(k_{i-1}\right) & \alpha_{4}\left(k_{i-1}\right) & \alpha_{5}\left(M_{t, i}, k_{i}, k_{i-1}\right) & \alpha_{6}\left(M_{t, i}, k_{i}, k_{i-1}\right) & \alpha_{3}\left(k_{i}\right) & \alpha_{4}\left(k_{i}\right)
\end{array}\right]\left[\begin{array}{c}
\lambda_{1}^{i-1} \\
\lambda_{2}^{i-1} \\
\lambda_{1}^{i} \\
\lambda_{2}^{i} \\
\lambda_{1}^{i+1} \\
\lambda_{2}^{i+1}
\end{array}\right]=\left[\begin{array}{l}
0 \\
0 \\
0 \\
0 \\
0 \\
0
\end{array}\right],
$$

with

$$
\begin{aligned}
& \alpha_{1}=\sin (\beta L)+\sinh (\beta L), \\
& \alpha_{2}=\cos (\beta L)+\cosh (\beta L), \\
& \alpha_{3}\left(k_{i}\right)=k_{i}(\sinh (\beta L)-\sin (\beta L)), \\
& \alpha_{4}\left(k_{i}\right)=k_{i}(\cosh (\beta L)-\cos (\beta L)), \\
& \alpha_{5}\left(M_{t, i}, k_{i}, k_{i-1}\right) \\
& =\left(k_{i}+k_{i-1}-M_{t, i} \omega_{n}^{2}\right)(\sin (\beta L)-\sinh (\beta L)) \\
& \quad+Y I \beta^{3}(\cos (\beta L)+\cosh (\beta L)), \\
& \alpha_{6}\left(M_{t, i}, k_{i}, k_{i-1}\right) \\
& =\left(k_{i}+k_{i-1}-M_{t, i} \omega_{n}^{2}\right)(\cos (\beta L)-\cosh (\beta L)) \\
& \quad+Y I \beta^{3}(\sinh (\beta L)-\sin (\beta L)) .
\end{aligned}
$$

$$
\left[\begin{array}{cccc}
0 & 0 & \alpha_{1} & \alpha_{2} \\
\alpha_{3}\left(k_{N-1}\right) & \alpha_{4}\left(k_{N-1}\right) & \alpha_{5}\left(M_{t, N}, 0, k_{N-1}\right) & \alpha_{6}\left(M_{t, N}, 0, k_{N-1}\right)
\end{array}\right]\left[\begin{array}{c}
\lambda_{1}^{N-1} \\
\lambda_{2}^{N-1} \\
\lambda_{1}^{N} \\
\lambda_{2}^{N}
\end{array}\right]=\left[\begin{array}{l}
0 \\
0 \\
0 \\
0
\end{array}\right] .
$$

Coupling all equations yields a system of $2 \times N$ equations, which can be written as the following matrix equation:

$$
\mathrm{C} \boldsymbol{\lambda}=\mathbf{0},
$$

where $\mathbf{C}$ is a coefficient matrix and $\lambda=\left[\lambda_{1}^{1}, \lambda_{2}^{1}, \lambda_{1}^{2}, \lambda_{2}^{2}, \ldots\right.$, $\left.\lambda_{1}^{N}, \lambda_{2}^{N}\right]^{T}$. This equation can have nonzero solution for $\lambda$ only if the determinant of the coefficient matrix vanishes (i.e., the coefficient matrix is singular). Setting the determinant of $\mathbf{C}$ equal to zero yields the characteristic equation that is satisfied by an infinite number of choices for $\beta$, denoted by $\beta_{r}$. The vector of unknowns $\lambda$ is calculated by replacing $\beta_{r}$ in the coefficient matrix $\mathbf{C}$ and then computing its null space. For each value of $\beta_{r}$ a different vector $\lambda_{r}$ is obtained.
Equation (7) can be obtained for each of the $N$ beams in the structure. Particular cases are the first and last beams. In the case of the first beam there is no previous neighbor; thus the following equation is obtained:

$$
\begin{gathered}
{\left[\begin{array}{cccc}
\alpha_{1} & \alpha_{2} & 0 & 0 \\
\alpha_{5}\left(M_{t, 1}, k_{1}, 0\right) & \alpha_{6}\left(M_{t, 1}, k_{1}, 0\right) & \alpha_{3}\left(k_{1}\right) & \alpha_{4}\left(k_{1}\right)
\end{array}\right]\left[\begin{array}{l}
\lambda_{1}^{1} \\
\lambda_{2}^{1} \\
\lambda_{1}^{2} \\
\lambda_{2}^{2}
\end{array}\right]} \\
=\left[\begin{array}{l}
0 \\
0 \\
0 \\
0
\end{array}\right],
\end{gathered}
$$

whereas for the last beam there is no next neighbor and the resulting equations are

Given $\beta_{r}$ and $\lambda_{r}$, the mode shapes are given by

$$
\begin{aligned}
\phi_{r, i}(x)= & \lambda_{r, 1}^{i}\left[\sin \left(\beta_{r} x\right)-\sinh \left(\beta_{r} x\right)\right] \\
& +\lambda_{r, 2}^{i}\left[\cos \left(\beta_{r} x\right)-\cosh \left(\beta_{r} x\right)\right] .
\end{aligned}
$$

The undamped natural frequency associated with the $r$ th mode is given by

$$
\omega_{n, r}=\beta_{r}^{2} \sqrt{\frac{Y I}{m}},
$$

where $m$ is the mass per unit length. As an example, Figure 3 illustrates the vibration modes of three elastically connected 
$\beta_{r} L=1.536$

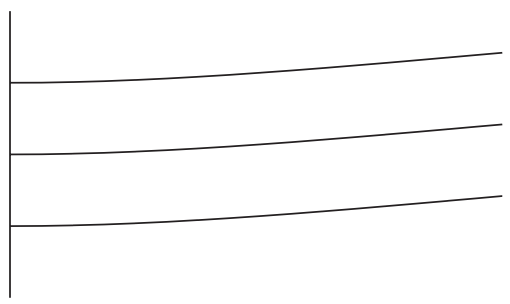

(a) Mode 1

$\beta_{r} L=4.192$

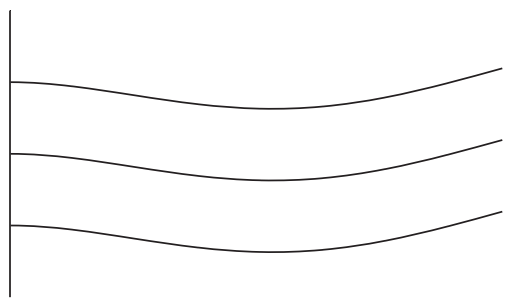

(d) Mode 4

$\beta_{r} L=7.254$

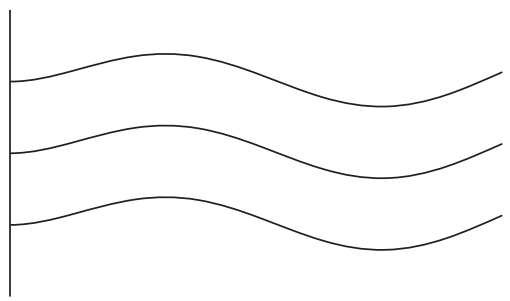

(g) Mode 7 $\beta_{r} L=1.573$

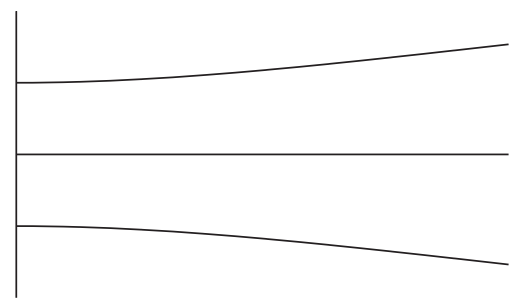

(b) Mode 2

$\beta_{r} L=4.193$

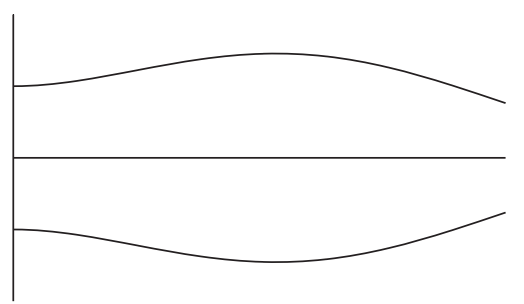

(e) Mode 5

$\beta_{r} L=7.254$

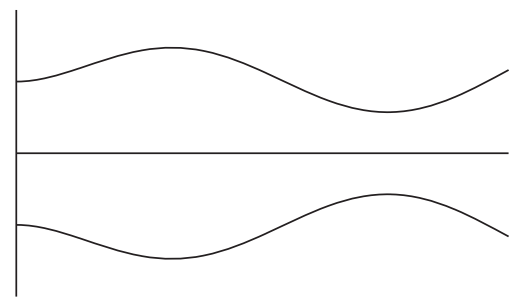

(h) Mode 8 $\beta_{r} L=1.640$

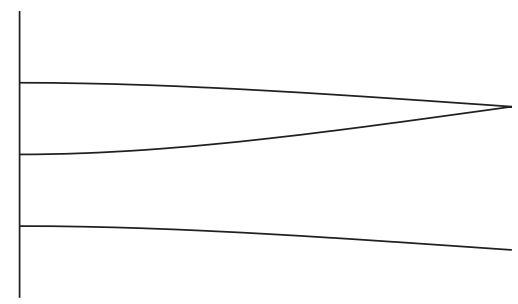

(c) Mode 3

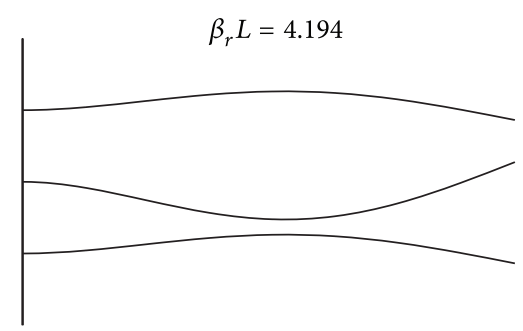

(f) Mode 6

$\beta_{r} L=7.254$

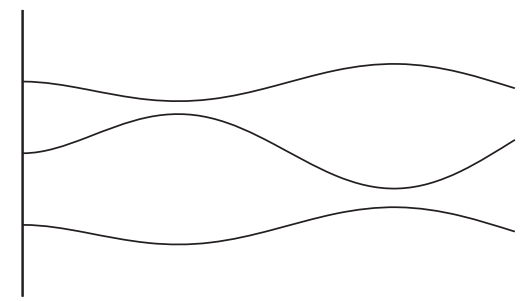

(i) Mode 9

FIGURE 3: First nine vibration modes of three elastically connected beams with identical tip masses and spring constants $\left(M_{t, i=1, \ldots, 3}=\right.$ $\left.0.3 m L, k_{1,2}=0.3 E I / L^{3}\right)$.

beams. In this example, all beams have a mass tip of $0.3 m L$, and all springs have a stiffness of $0.3 Y I / L^{3}$.

\section{Electromechanical Model of a Bimorph Piezoelectric Beam}

3.1. Mechanical Equation. Let us consider the bimorph cantilever beam shown in Figure 4. The bimorph beam consists in two layers of piezoelectric material (PZT) bonded to a substructure layer. Assuming an Euler-Bernoulli beam, the equation of motion can be written as [33]

$$
\begin{aligned}
& \frac{\partial^{2} M(x, t)}{\partial x^{2}}+c_{s} I \frac{\partial^{5} w_{\text {rel }}(x, t)}{\partial x^{4} \partial t}+c_{a} \frac{\partial w_{\text {rel }}(x, t)}{\partial t} \\
& \quad+m \frac{\partial^{2} w_{\text {rel }}(x, t)}{\partial t^{2}} \\
& =-\left[m+M_{t} \delta(x-L)\right] \frac{\partial^{2} w_{b}(t)}{\partial t^{2}}-c_{a} \frac{\partial w_{b}(t)}{\partial t},
\end{aligned}
$$

where $w_{\text {rel }}(x, t)$ is the transverse deflection of the beam relative to the base, $w_{b}(t)$ is the base displacement, $M(x, t)$ is the internal bending moment, $c_{s}$ is the equivalent coefficient of strain rate damping, $c_{a}$ is the viscous air damping, $I$ is the area moment of inertia of the cross section, $m$ is the mass per unit length, $M_{t}$ is the tip mass, and $\delta(x)$ is the Dirac delta function. 
The internal moment is given by the integral of the stress distribution over the cross section of the beam shown in

$$
\begin{aligned}
& M(x, t)=-b\left[\int_{-h_{p}-h_{s} / 2}^{-h_{s} / 2} T^{p} y d y+\int_{-h_{s} / 2}^{h_{s} / 2} T^{s} y d y\right. \\
& \left.+\int_{h_{s} / 2}^{h_{s} / 2+h_{p}} T^{p} y d y\right],
\end{aligned}
$$

where $b$ is the width of the beam, $h_{p}$ is the thickness of each PZT layer, $h_{s}$ is the thickness of the substructure (see Figure 4), and $T$ is the stress. Superscripts $p$ and $s$ stand for PZT and substructure, respectively. The stress distribution across the section is given by the strain-stress relations of the substructure and PZT layers, which are given by

$$
\begin{aligned}
& T^{s}=Y^{s} S^{s}, \\
& T^{p}=Y^{p} S^{p}-d_{31} Y^{p} E,
\end{aligned}
$$

where $S$ is the strain, $Y$ is Young's modulus, $d$ is the piezoelectric constant, and $E$ is the electric field. The bending strain can be expressed in terms of the radius of curvature as

$$
S(x, y, t)=-y \frac{\partial^{2} w_{\text {rel }}(x, t)}{\partial x^{2}}
$$

Since the piezoelectric layers are connected in parallel they have the same voltage, but the instantaneous electric fields are in opposite directions. The electric field can be written in terms of the voltage across the PZT, $v(t)$, and the thickness of the PZT layer, $h_{p}$, by the following equation:

$$
\begin{aligned}
& E_{1}(t)=-\frac{v(t)}{h_{p}} \text { in the top layer, } \\
& E_{2}(t)=\frac{v(t)}{h_{p}} \text { in the bottom layer. }
\end{aligned}
$$

Replacing (16) to (19) in (15), we obtain

$$
\begin{aligned}
M(x, t)= & \frac{2 b}{3}\left[Y^{s} \frac{h_{s}^{3}}{12}+Y^{p}\left(\left(h_{p}+\frac{h_{s}}{2}\right)^{3}-\frac{h_{s}^{3}}{8}\right)\right] \\
& \cdot \frac{\partial^{2} w_{\mathrm{rel}}(x, t)}{\partial x^{2}} \\
& +\frac{d_{31} Y^{p} b}{h_{p}}\left[\frac{h_{s}^{2}}{4}-\left(h_{p}+\frac{h_{s}}{2}\right)^{2}\right] v(t) .
\end{aligned}
$$

Equation (20) can be expressed as

$$
\begin{aligned}
M(x, t)= & Y I \frac{\partial^{2} w_{\text {rel }}(x, t)}{\partial x^{2}} \\
& +\vartheta v(t)[\Phi(x)-\Phi(x-L)],
\end{aligned}
$$

where $L$ is the length of the beam and $\Phi$ the Heaviside function. It has been assumed that the PZT layer covers the entire length of the beam. The constant $Y I$ represents the bending stiffness of the beam and is given by

$$
Y I=\frac{2 b}{3}\left[Y^{s} \frac{h_{s}^{3}}{12}+Y^{p}\left(\left(h_{p}+\frac{h_{s}}{2}\right)^{3}-\frac{h_{s}^{3}}{8}\right)\right],
$$

and the term $\vartheta$ represents the coupling between the strain and the voltage and is given by

$$
\vartheta=\frac{d_{31} Y^{p} b}{h_{p}}\left[\frac{h_{s}^{2}}{4}-\left(h_{p}+\frac{h_{s}}{2}\right)^{2}\right] .
$$

Substituting (21) in (14), we obtain the mechanical equation of motion with electrical coupling:

$$
\begin{aligned}
Y I \frac{\partial^{4} w_{\text {rel }}(x, t)}{\partial x^{4}}+c_{s} I \frac{\partial^{5} w_{\text {rel }}(x, t)}{\partial x^{4} \partial t}+c_{a} \frac{\partial w_{\text {rel }}(x, t)}{\partial t} \\
\quad+m \frac{\partial^{2} w_{\text {rel }}(x, t)}{\partial t^{2}} \\
\quad+\vartheta v(t)\left[\frac{\partial \delta(x)}{\partial x}-\frac{\partial \delta(x-L)}{\partial x}\right] \\
=-\left[m+M_{t} \delta(x-L)\right] \frac{\partial^{2} w_{b}(t)}{\partial t^{2}}-c_{a} \frac{\partial w_{b}(t)}{\partial t} .
\end{aligned}
$$

3.2. Electrical Equation. To derive the electrical equation let us start with the piezoelectric constitutive relation given in

$$
D=d_{31} T^{p}+\varepsilon_{33}^{T} E
$$

where $D$ is the electric displacement and $\varepsilon_{33}^{T}$ is the permittivity at constant stress. Replacing the definition of $T^{p}$ from (17) yields

$$
\begin{aligned}
D(x, t) & =d_{31}\left(Y^{p} S^{p}-d_{31} E\right)+\varepsilon_{33}^{T} E \\
& =d_{31} Y^{p} S^{p}+E\left(\varepsilon_{33}^{T}-d_{31}^{2} Y^{p}\right) \\
& =d_{31} Y^{p} S^{p}-\varepsilon_{33} \frac{v(t)}{h_{p}},
\end{aligned}
$$

with $\varepsilon_{33}^{S}=d_{31}^{2} Y^{p}-\varepsilon_{33}^{T}$. The strain $S^{p}$ evaluated at the middle of the PZT layer is given by

$$
S^{p}=-h_{p c} \frac{\partial^{2} w_{\mathrm{rel}}(x, t)}{\partial x^{2}},
$$

where $h_{p c}=h_{s} / 2+h_{p} / 2$ is the distance of the center of the PZT layer to the neural axis. Thus,

$$
D(x, t)=-d_{31} Y^{p} h_{p c} \frac{\partial^{2} w_{\text {rel }}(x, t)}{\partial x^{2}}-\varepsilon_{33}^{S} \frac{v(t)}{h_{p}} .
$$

The electric charge is obtained by integrating the electric displacement over the area of the beam. The resulting expression is

$$
q(t)=-\int_{0}^{L}\left(d_{31} Y^{p} h_{p c} \frac{\partial^{2} w_{\text {rel }}(x, t)}{\partial x^{2}}+\varepsilon_{33}^{s} \frac{v(t)}{h_{p}}\right) b d x .
$$


Therefore the current generated by one PZT layer is

$$
\begin{aligned}
i(t)= & \frac{\partial q(t)}{\partial t} \\
= & -\frac{\varepsilon_{33}^{S} b L}{h_{p}} \frac{\partial v(t)}{\partial t} \\
& -\int_{0}^{L}\left(d_{31} Y^{p} h_{p c} b \frac{\partial^{3} w_{\text {rel }}(x, t)}{\partial x^{2} \partial t}\right) d x .
\end{aligned}
$$

Since both layers are connected in parallel, the current generated by the bimorph beam is given by

$$
\begin{aligned}
i(t)= & i_{1}(t)+i_{2}(t) \\
= & -2 \frac{\varepsilon_{33}^{S} b L}{h_{p}} \frac{\partial v(t)}{\partial t} \\
& -2 \int_{0}^{L}\left(d_{31} Y^{p} h_{p c} b \frac{\partial^{3} w_{\text {rel }}(x, t)}{\partial x^{2} \partial t}\right) d x .
\end{aligned}
$$

If the beam is connected directly to a resistive load, the voltage is given by $v(t)=R_{l} i(t)$. Substituting this relation in (31) yields

$$
\begin{aligned}
v(t) & =-2 R_{l}\left[\frac{\varepsilon_{33}^{S} b L}{h_{p}} \frac{\partial v(t)}{\partial t}\right. \\
& \left.+\int_{0}^{L}\left(d_{31} Y^{p} h_{p c} b \frac{\partial^{3} w_{\text {rel }}(x, t)}{\partial x^{2} \partial t}\right) d x\right] .
\end{aligned}
$$

The electrical equation with mechanical coupling is obtained by rearranging (32):

$$
\begin{aligned}
\frac{v(t)}{2 R_{l}} & +\frac{\varepsilon_{33}^{S} b L}{h_{p}} \frac{\partial v(t)}{\partial t} \\
= & -\int_{0}^{L}\left(d_{31} Y^{p} h_{p c} b \frac{\partial^{3} w_{\text {rel }}(x, t)}{\partial x^{2} \partial t}\right) d x
\end{aligned}
$$

\section{Voltage and Power Output of an Array of Piezoelectric Beams Connected by Springs}

Equations (24) and (33) are the electromechanical equations for a single bimorph beam under transverse vibrations. Let us consider the case of multiple beams connected by springs with a parallel electrical connection, as shown in Figure 5. In that case the mechanical equation of motion is given by

$$
\begin{aligned}
Y I \frac{\partial^{4} w_{\text {rel }}(x, t)}{\partial x^{4}}+c_{s} I \frac{\partial^{5} w_{\text {rel }}(x, t)}{\partial x^{4} \partial t}+c_{a} \frac{\partial w_{\text {rel }}(x, t)}{\partial t} \\
+m \frac{\partial^{2} w_{\text {rel }}(x, t)}{\partial t^{2}} \\
+\vartheta \sum_{i=1}^{N} v_{i}(t)\left[\frac{\partial \delta(x-(i-1) L)}{\partial x}-\frac{\partial \delta(x-i L)}{\partial x}\right] \\
=-\left[m+\sum_{i=1}^{N} M_{t, i} \delta(x-i L)\right] \frac{\partial^{2} w_{b}(t)}{\partial t^{2}}-c_{a} \frac{\partial w_{b}(t)}{\partial t},
\end{aligned}
$$

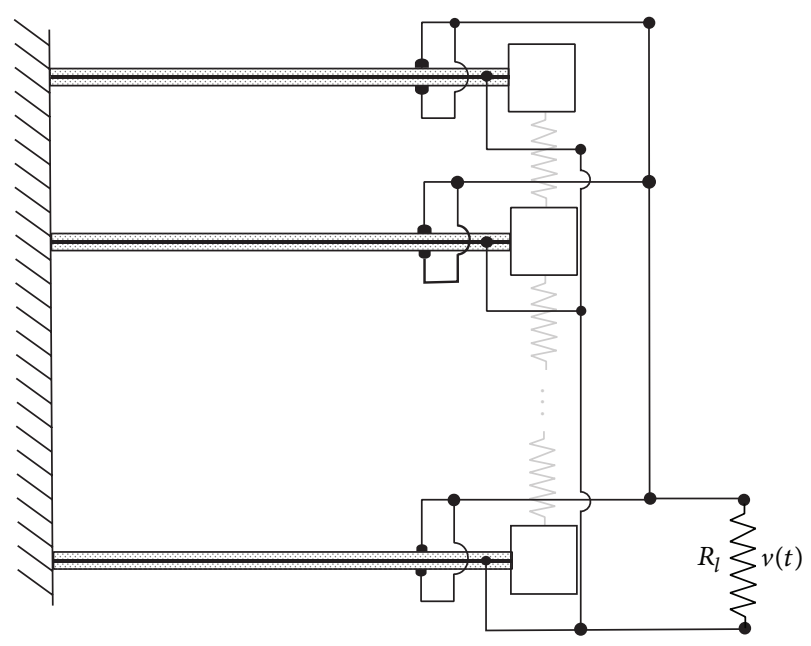

FIGURE 5: A parallel electrical connection of an array of bimorph beams connected by springs.

where $v_{i}(t)$ is the voltage of the $i$ th beam. Since the beams are electrically connected in parallel they have the same voltage; thus $v_{1}(t)=v_{2}(t)=\cdots=v_{n}(t)=v(t)$. It has been assumed that all the beams are identical except for the tip mass. It should be noted that the springs are included as boundary conditions. Therefore, they affect the vibration modes, but not the equation of motion.

The solution for the relative motion of the system can be represented by a sum of its mode shapes as

$$
w_{\text {rel }}(x, t)=\sum_{r} \phi_{r}(x) \eta_{r}(t),
$$

where $\phi_{r}(x)$ and $\eta_{r}(t)$ are the $r$ th mode shape and modal coordinate, respectively. The $r$ th mode shape can be defined by segments as

$$
\phi_{r}(x)
$$

$$
= \begin{cases}\phi_{r, 1}(x) & 0 \leq x \leq L \\ \phi_{r, 2}(x-L) & L \leq x \leq 2 L \\ \vdots & \vdots \\ \phi_{r, N}(x-(N-1) L) & (N-1) L \leq x \leq N L,\end{cases}
$$

where $\phi_{r, i}$ is the $r$ th mode shape of the $i$ th beam, $L$ is the length of one beam, and $N$ is the number of beams.

Replacing (35), the mechanical equation of motion can be written as

$$
\begin{gathered}
{\left[\omega_{r}^{2} \eta_{r}(t)+2 \xi_{r} \omega_{r} \dot{\eta}_{r}(t)+\ddot{\eta}_{r}(t)\right] \alpha_{r}+\sum_{i=1}^{N} \chi_{r, i} v(t)} \\
=\left[-m \frac{\partial^{2} w_{b}(x, t)}{\partial t^{2}}-c_{a} \frac{\partial w_{b}(x, t)}{\partial t}\right] \gamma_{r} \\
-\frac{\partial^{2} w_{b}(x, t)}{\partial t^{2}} \sum_{i=1}^{N} M_{t, i} \phi_{r}(i L),
\end{gathered}
$$


with

$$
\begin{aligned}
\chi_{r, i} & =\vartheta\left(-\phi_{r, i}(L)+\frac{\partial \phi_{r, i}(L)}{\partial x}\right), \\
\xi_{r} & =\frac{c_{s} I \omega_{r}}{2 Y I}+\frac{c_{a}}{2 m \omega_{r}}, \\
\alpha_{r} & =\int_{0}^{N L} m \phi_{r}^{2}(x) d x, \\
\gamma_{r} & =\int_{0}^{N L} \phi_{r}(x) d x .
\end{aligned}
$$

Assuming a harmonic base motion, $w_{b}(x, t)=Y_{0} e^{j \omega t}$, and a harmonic response, $\eta_{r}(t)=N_{r} e^{j \omega t}$ and $v(t)=V e^{j \omega t}$, (37) becomes

$$
\begin{gathered}
{\left[\omega_{n, r}^{2} N_{r}+2 j \xi_{r} \omega_{n, r} \omega N_{r}-\omega^{2} N_{r}\right] \alpha_{r}+V \sum_{i=1}^{N} \chi_{r, i}} \\
=\left(m \omega^{2}-j \omega c_{a}\right) Y_{0} \gamma_{r}+\omega^{2} Y_{0} \sum_{i=1}^{N} M_{t, i} \phi_{r}(i L) .
\end{gathered}
$$

Rearranging (39), the amplitude of the temporal term, $N_{r}$, can be written as follows:

$$
\begin{aligned}
& N_{r} \\
& =\frac{\left(m \omega^{2}-j \omega c_{a}\right) Y_{0} \gamma_{r}+\omega^{2} Y_{0} \sum_{i=1}^{N} M_{t, i} \phi_{r}(i L)-V \sum_{i=1}^{N} \chi_{r, i}}{\left(\omega_{r}^{2}+2 j \xi_{r} \omega_{r} \omega-\omega^{2}\right) \alpha_{r}} .
\end{aligned}
$$

For simplicity, (40) can be expressed as

$$
N_{r}=a_{r}-V \sum_{i=1}^{N} b_{r, i},
$$

with

$$
\begin{aligned}
a_{r} & =\frac{\left(m \omega^{2}-j \omega c_{a}\right) Y_{0} \gamma_{r}+\omega^{2} Y_{0} \sum_{i=1}^{N} M_{t, i} \phi_{r}(i L)}{\left(\omega_{r}^{2}+2 j \xi_{r} \omega_{r} \omega-\omega^{2}\right) \alpha_{r}}, \\
b_{r, i} & =\frac{\chi_{r, i}}{\left(\omega_{r}^{2}+2 j \xi_{r} \omega_{r} \omega-\omega^{2}\right) \alpha_{r}} .
\end{aligned}
$$

The electrical equation needs to be derived for each beam individually. The relative motion for the $i$ th beam is given by

$$
w_{\mathrm{rel}, i}(x, t)=\sum_{r} \phi_{r, i}(x) \eta_{r}(t) .
$$

Replacing (43) in (30) yields

$$
\begin{aligned}
i_{i}(t)= & -2 \frac{\varepsilon_{33}^{S} b L}{h_{p}} \frac{\partial v(t)}{\partial t} \\
& -\left.2 d_{31} Y^{p} h_{p c} b \sum_{r} \frac{\partial \phi_{r, i}(x)}{\partial x}\right|_{0} ^{L} \dot{\eta}_{r}(t) .
\end{aligned}
$$

If the beams are connected directly to a resistive load, the voltage is given by $v(t)=R_{l} i(t)=R_{l} \sum_{i} i_{i}(t)$; substituting this condition equation (44) becomes

$$
\begin{aligned}
\frac{v(t)}{2 R_{l}}= & -\frac{\varepsilon_{33}^{S} b L N}{h_{p}} \frac{\partial v(t)}{\partial t} \\
& -\left.d_{31} Y^{p} h_{p c} b \sum_{r} \sum_{i} \frac{\partial \phi_{r, i}(x)}{\partial x}\right|_{0} ^{L} \dot{\eta}_{r}(t) .
\end{aligned}
$$

Replacing the harmonic response condition in (45) yields

$$
\begin{aligned}
\frac{V}{2 R_{l}}= & -j \omega \frac{\varepsilon_{33}^{S} b L N}{h_{p}} V \\
& -\left.d_{31} Y^{p} h_{p c} b \sum_{r} \sum_{i} \frac{\partial \phi_{r, i}(x)}{\partial x}\right|_{0} ^{L} j \omega N_{r} .
\end{aligned}
$$

Rearranging (46), we obtain

$$
V \kappa_{c}=\sum_{r} \sum_{i} \varphi_{r, i} j \omega N_{r}
$$

with

$$
\begin{aligned}
\varphi_{r, i} & =-\left.\frac{d_{31} Y^{p} h_{p c} h_{p}}{\varepsilon_{33}^{S} L N} \frac{\partial \phi_{r, i}(x)}{\partial x}\right|_{0} ^{L}, \\
\kappa_{c} & =j \omega+\frac{h_{p}}{2 R_{l} \varepsilon_{33}^{S} b L N} .
\end{aligned}
$$

Replacing $N_{r}$ from (41) in (47) the following equation is obtained:

$$
V \kappa_{c}=\sum_{r} \sum_{i} \varphi_{r, i} j \omega\left(a_{r}-V \sum_{k=1}^{N} b_{r, k}\right) .
$$

Rearranging (49) the voltage amplitude can be written as shown in

$$
V=\frac{\sum_{r} \sum_{i} \varphi_{r, i} j \omega a_{r}}{\kappa_{c}+\sum_{r} \sum_{i} \varphi_{r, i} j \omega \sum_{k=1}^{N} b_{r, k}} .
$$

The voltage FRF is defined as the ratio of the voltage output to the base acceleration, $V /-\omega^{2} Y_{0}$. The instantaneous power output can be obtained from the relation $P(t)=$ $v^{2}(t) / R_{l}$. Therefore, the FRF of the power output is simply the square of the voltage FRF divided by $R_{l}$.

\section{Results}

5.1. Analytical. This section presents the results of the power FRF for different number of beams and different combinations of tip masses and spring constants in the array. The geometric, material, and electromechanical parameters of the beams are given in Table 1. These are the parameters of the experimental beams used in the next section.

The voltage is computed by (50) and the power FRF is computed as $V^{2} /\left[R_{l}\left(\omega^{2} Y_{0}\right)^{2}\right]$. The value of the external load 


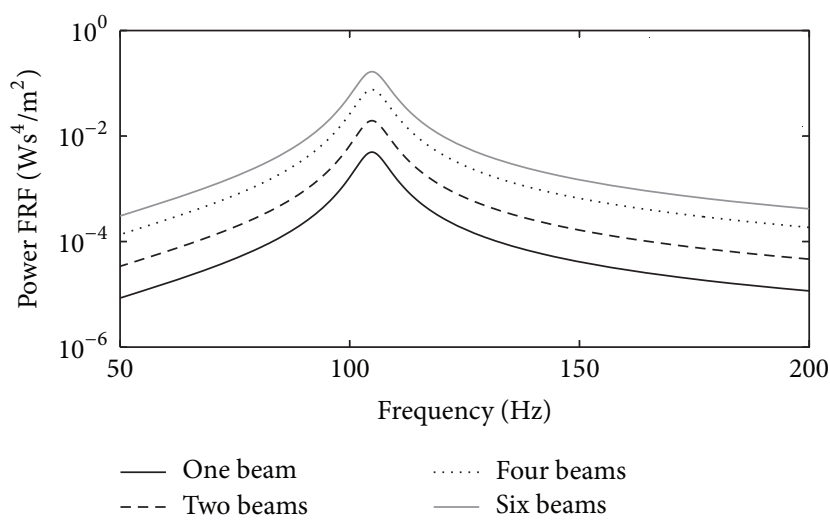

(a) Connected

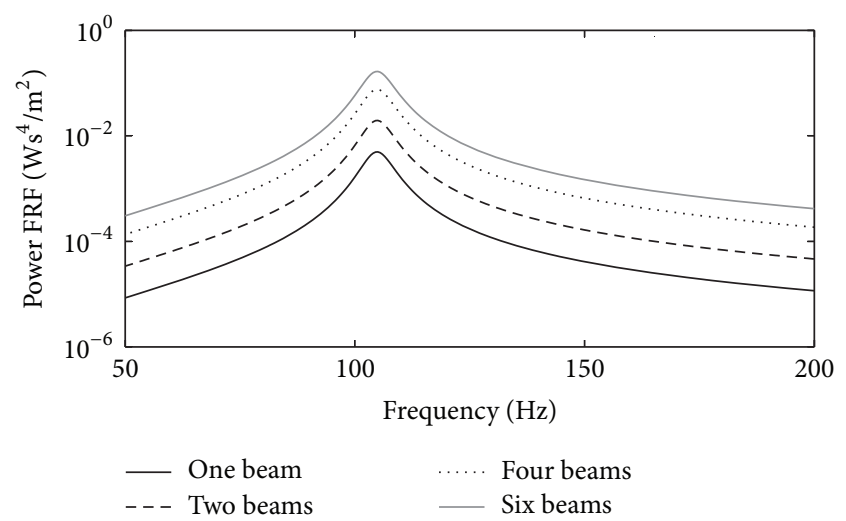

(b) Disconnected

FIGURE 6: Power FRF with an increasing number of identical bimorph beams, $M_{t, i=1, \ldots, 6}=0.3 m L$ and $k_{i=1, \ldots, 5}=0.3 Y I / L^{3}$.

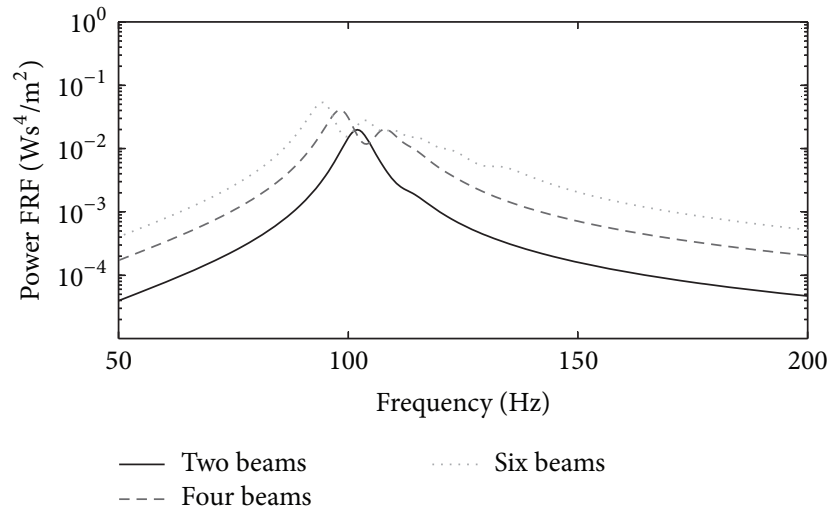

(a) Connected

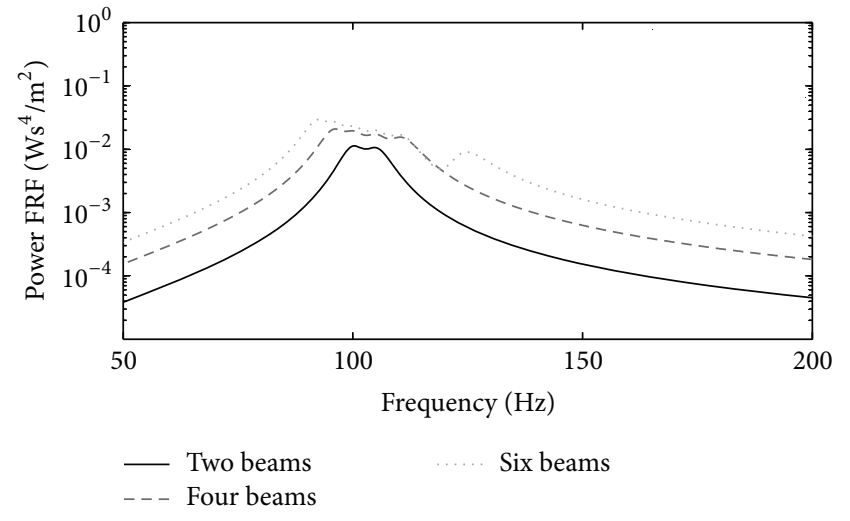

(b) Disconnected

FIgURE 7: Power FRF with an increasing number of bimorph beams with different tip masses. Two beams: $M_{t, 1}=0.3 m L, M_{t, 2}=0.35 m L$, $k=0.3 Y I / L^{3}$; three beams: $M_{t, 1}=0.25 m L, M_{t, 2}=0.30 m L, M_{t, 3}=0.35 m L, k_{1,2}=0.3 Y I / L^{3}$; six beams: $M_{t, 1}=0.15 m L, M_{t, 2}=0.20 m L$, $M_{t, 3}=0.30 m L, M_{t, 4}=0.35 m L, M_{t, 5}=0.40 m L, M_{t, 6}=0.45 m L, k_{i=1, \ldots, 5}=0.3 Y I / L^{3}$.

TABle 1: Parameters of the bimorph beams.

\begin{tabular}{lc}
\hline Length of the beam, $L(\mathrm{~mm})$ & 48.2 \\
Width of the beam, $b(\mathrm{~mm})$ & 10 \\
Thickness of the substructure, $h_{s}(\mathrm{~mm})$ & 0.19 \\
Thickness of the PZT, $h_{p}(\mathrm{~mm})$ & 0.24 \\
Young's modulus of the substructure, $Y_{s}(\mathrm{GPa})$ & 40.4 \\
Young's modulus of the PZT, $Y_{p}(\mathrm{GPa})$ & 55 \\
Mass density of the substructure, $\rho_{s}\left(\mathrm{~kg} / \mathrm{m}^{3}\right)$ & 1519 \\
Mass density of the PZT, $\rho_{p}\left(\mathrm{~kg} / \mathrm{m}^{3}\right)$ & 8140 \\
Piezoelectric constant, $d_{31}(\mathrm{pm} / \mathrm{V})$ & -180 \\
Permittivity, $\varepsilon_{33}^{s}(\mathrm{nF} / \mathrm{m})$ & 13.28 \\
Strain rate damping, $c_{s} I\left(\mathrm{kgm}{ }^{4} / \mathrm{s}\right)$ & $1.16 \times 10^{-6}$ \\
Viscous air damping, $c_{a}(\mathrm{~kg} / \mathrm{s})$ & 0.4 \\
\hline
\end{tabular}

resistance is defined for each case as the optimum resistor that maximizes the power output. To study the advantages and disadvantages of the proposed system, the results are compared with those obtained by an array of disconnected beams (with no springs).
First, the case of identical beams is investigated; the results are shown in Figure 6(a). It is obtained that, in the case of identical beams, the only modes that are excited are the modes where all beams move in phase, such as modes 1, 4, and 7 in Figure 3. In this case, it is also found that the natural frequencies do not depend on the spring stiffness because all beams are in phase and the springs do not deform. Furthermore, the results are exactly the same as the ones obtained with disconnected beams, as illustrated in Figure 6(b).

In the case of beams with different tip masses, the results of the FRF power do depend on the springs connecting the beams, as shown in Figure 7. If the beams are disconnected and new beams with different tip masses are added to the array, then the frequency band of the device is increased obtaining a similar power amplitude for all resonant frequencies. On the other hand, if the beams are connected by spring and new beams are included in the array, then the frequency band is increased, but the amplitude related to each resonant frequency varies.

For an array of piezoelectric beams connected by springs, the frequency band of operation and the power amplitude 


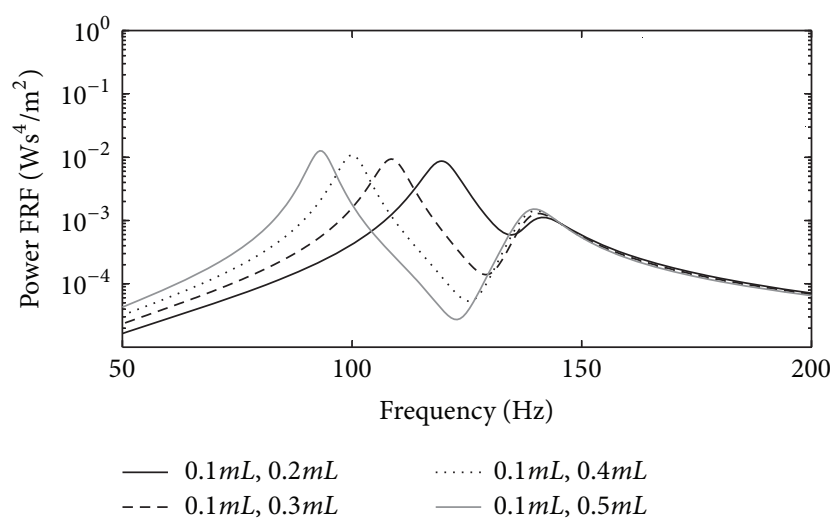

(a) Varying tip masses $\left(k=0.3 Y I / L^{3}\right)$

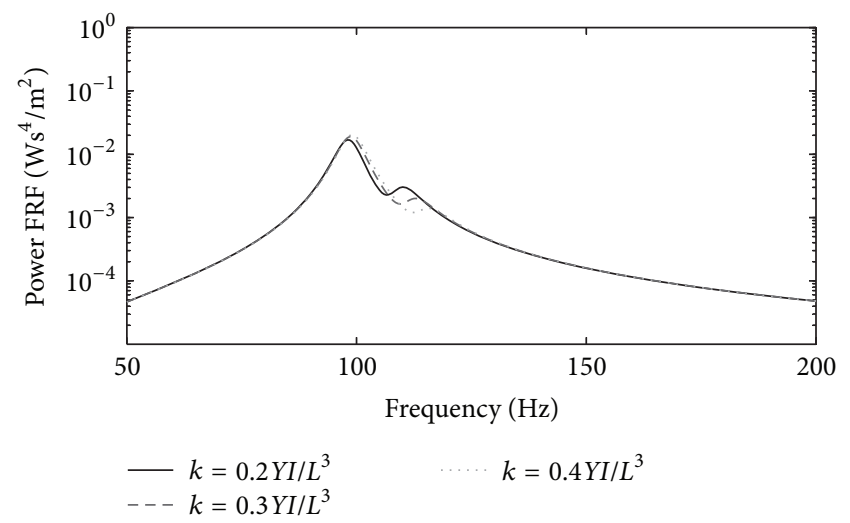

(b) Varying spring constants $\left(M_{t, 1}=0.3 m L, M_{t, 2}=0.4 m L\right)$

FIGURE 8: Power FRF of two bimorph beams with different combinations of tip masses and spring constants.

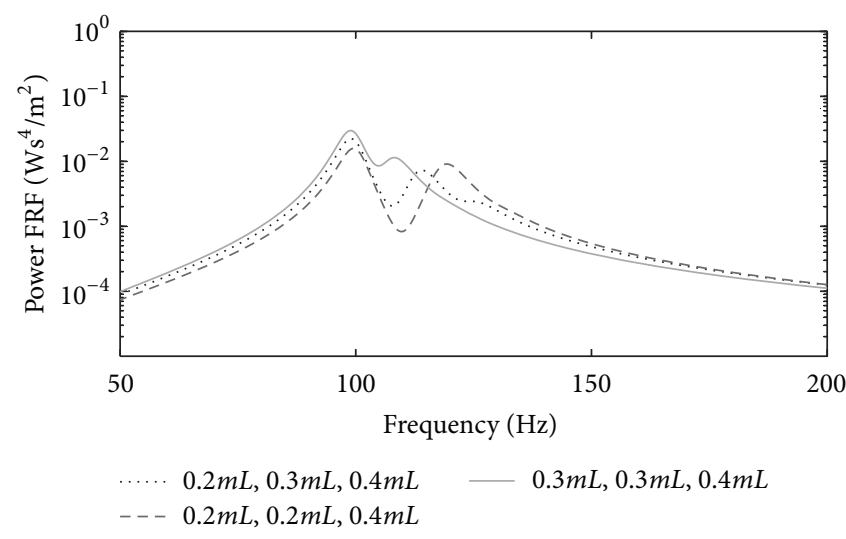

(a) Varying tip masses $\left(k=0.3 Y I / L^{3}\right)$

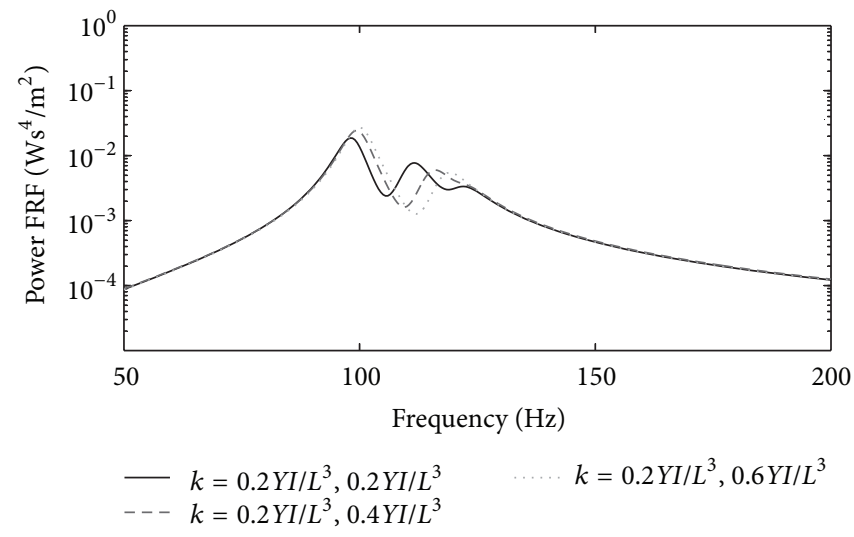

(b) Varying spring constants $\left(M_{t, 1}=0.2 m L, M_{t, 2}=0.3 m L, M_{t, 3}=\right.$ $0.4 m L)$

FIGURE 9: Power FRF of three bimorph beams with different combinations of tip masses and spring constants.

associated with each resonant frequency depend on the combination of tip masses and springs constants. This is more evident in Figures 8 and 9, where different combinations of springs and tip masses are studied for the case of two and three beams. In general, increasing the tip mass of the beams decreases some of the resonant frequencies and increasing the spring stiffness increases the frequency band.

To compare the performances of an array of beams connected by springs and one of the disconnected beams, a case of ten beams with both situations is plotted in Figure 10. It is possible to see that the array with connected beams has a slightly wider frequency band, which increases with an increment of the springs constants. Furthermore, by comparing the areas below both curves in the frequency range $50-200 \mathrm{~Hz}$, the array with connected beams provides an area between $20 \%$ and 36\% larger than the array with disconnected beams. Thus, by connecting the beams, the performance of the array can be improved. It should be noted that the previous results are obtained with arbitrary values for the tip masses and spring constants. Therefore, this performance can be further improved by a proper design.
5.2. Experimental. To validate the proposed model a preliminary experimental campaign was performed. Figure 11 presents the experimental setup. It consists of a signal generator connected to a power amplifier and to an electrodynamic shaker, which produces a controlled base vibration. An array of three piezoelectric bimorph beams is mounted over the shaker; Figure 12 shows this array. The cantilever bimorph beams have a tip mass at the free-end and can be connected with springs between them. Three experiments were performed. In the first case, no springs were used and each beam was connected independently to a $1 \mathrm{k} \Omega$ resistive load; in this case the voltage generated by each beam is monitored individually. In the second case, no springs were used and the beams were electrically connected in parallel to a resistive load of $1 \mathrm{k} \Omega$. In the last case, the beams were connected with springs between them and they were electrically connected in parallel to a resistive load of $1 \mathrm{k} \Omega$.

The properties of the bimorph beams are the ones given in Table 1; the tip masses are $M_{t, 1}=6.5 \mathrm{~mL}, M_{t, 2}=3.5 \mathrm{~mL}$, $M_{t, 3}=1.9 \mathrm{~mL}$ and the spring constants are $k_{1}=k_{2}=$ $2.92 Y I / L^{3}$. 


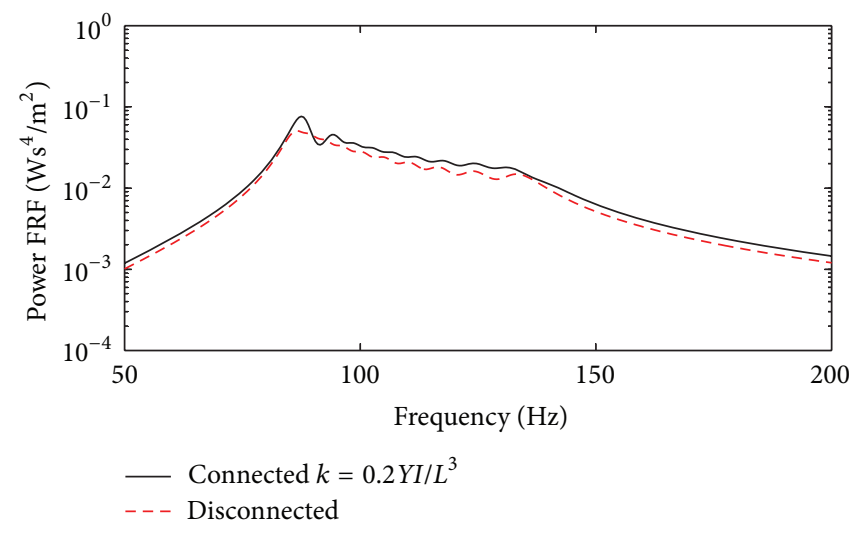

(a) $k_{i=1, \ldots, 9}=0.2 Y I / L^{3}$

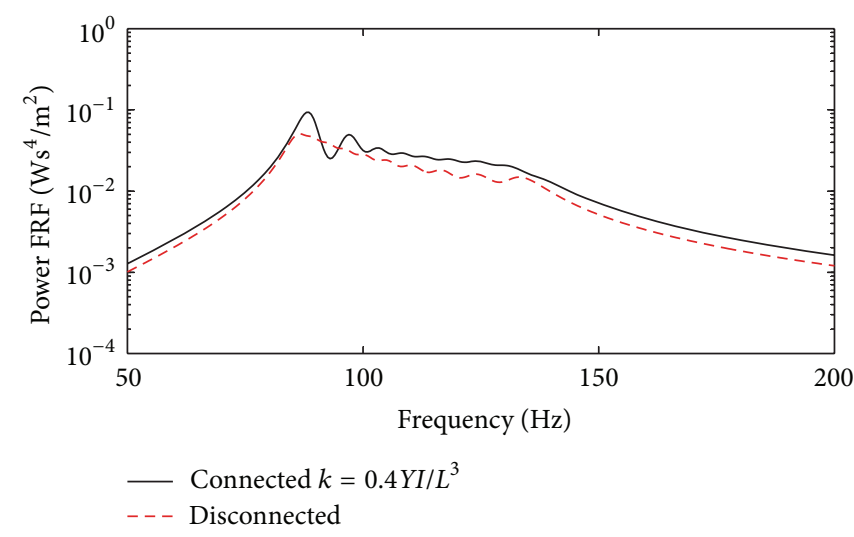

(c) $k_{i=1, \ldots, 9}=0.4 Y I / L^{3}$

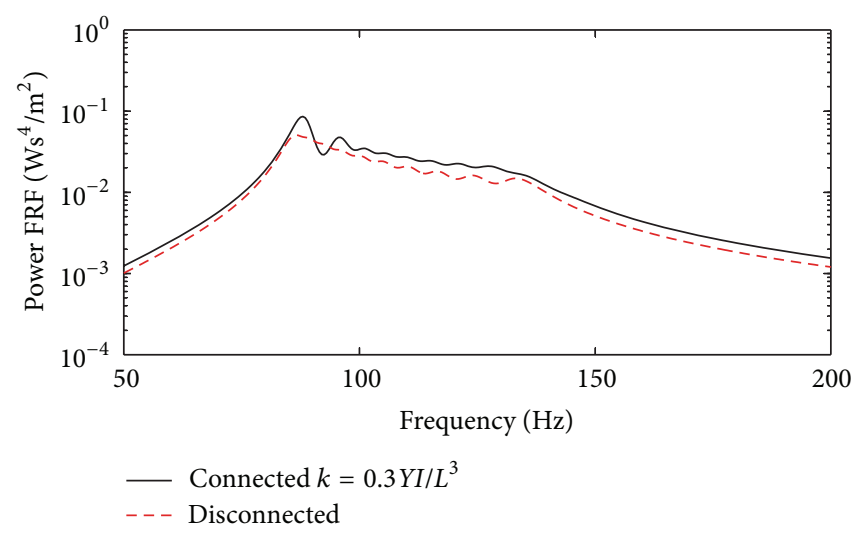

(b) $k_{i=1, \ldots, 9}=0.3 Y I / L^{3}$

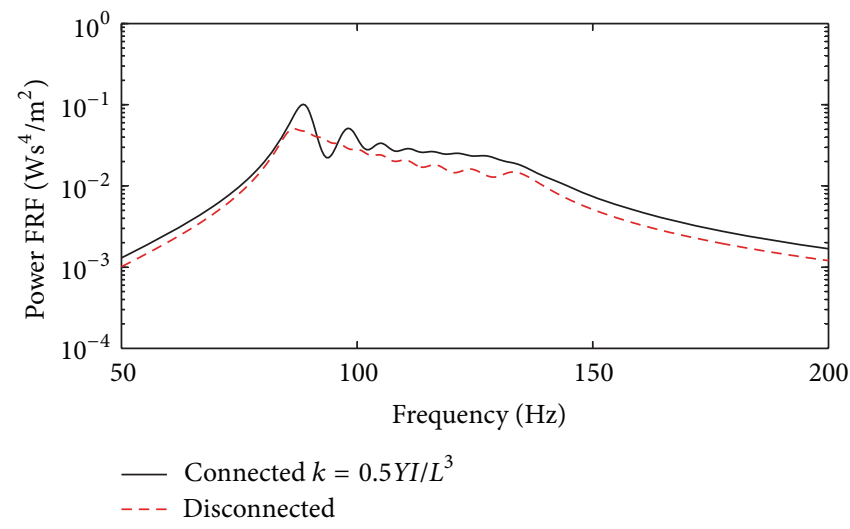

(d) $k_{i=1, \ldots, 9}=0.5 Y I / L^{3}$

Figure 10: Power FRF with ten bimorph beams: $M_{t, 1}=0.1 m L, M_{t, 2}=0.15 m L, M_{t, 3}=0.2 m L, M_{t, 4}=0.25 m L, M_{t, 5}=0.3 m L, M_{t, 6}=0.35 m L$, $M_{t, 7}=0.4 m L, M_{t, 8}=0.45 m L, M_{t, 9}=0.5 m L, M_{t, 10}=0.55 m L$.

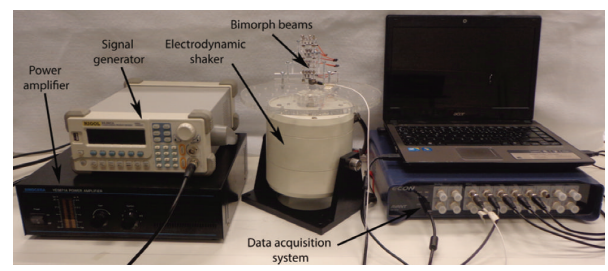

FIGURE 11: Experimental setup.

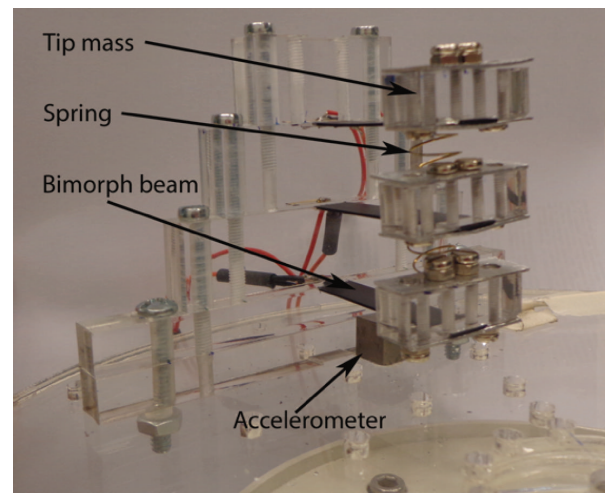

FIGURE 12: Experimental bimorph beams.
During the three experiments the system is subjected to a harmonic base displacement with frequencies in the range $20-70 \mathrm{~Hz}$. The results for the first experiment are presented in Figure 13(a) and are compared with the ones obtained by an analytical model in Figure 13(b). Both, analytical and experimental, curves are very similar. The only difference is that the experimental power increases from beams 1 to 3 , and the analytical model predicted the opposite behavior. This unexpected behavior seems to be related to a resonance of the supporting structure; in the three experimental curves, it is possible to see an increment of the power near $60 \mathrm{~Hz}$, which can be explained by a resonance of the structure.

Figure 14 presents the experimental and analytical results for the second and third experiments. It is possible to see a good agreement between the experimental and analytical curves, although, similarly to the results in Figure 13(a), there is an increment of the power near $60 \mathrm{~Hz}$ that confirms a structural resonance.

Comparing the areas below both experimental curves in Figure 13(b) in the frequency range $20-70 \mathrm{~Hz}$, the array with connected beams provides an area 5\% larger than the array with disconnected beams, which confirms that the addition of springs can increment the generated power. These results can be improved by using softer springs, which have lower energy losses, and by solving the structural resonance problem. 


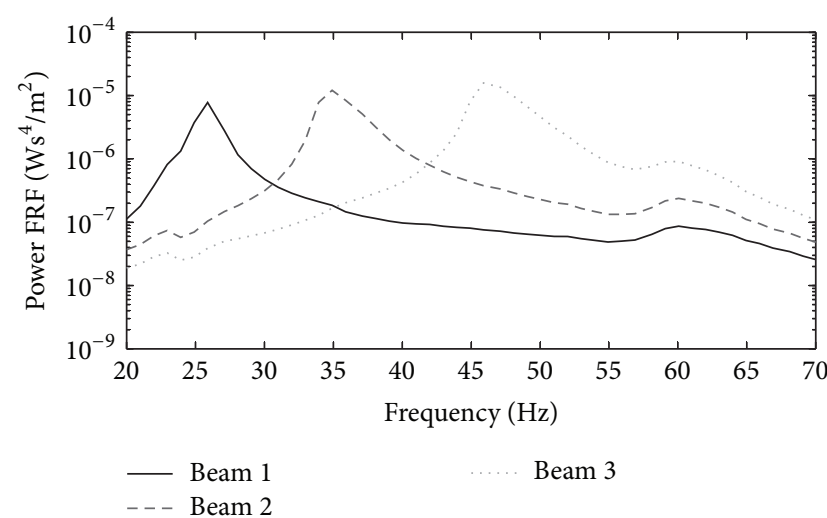

(a) Experimental

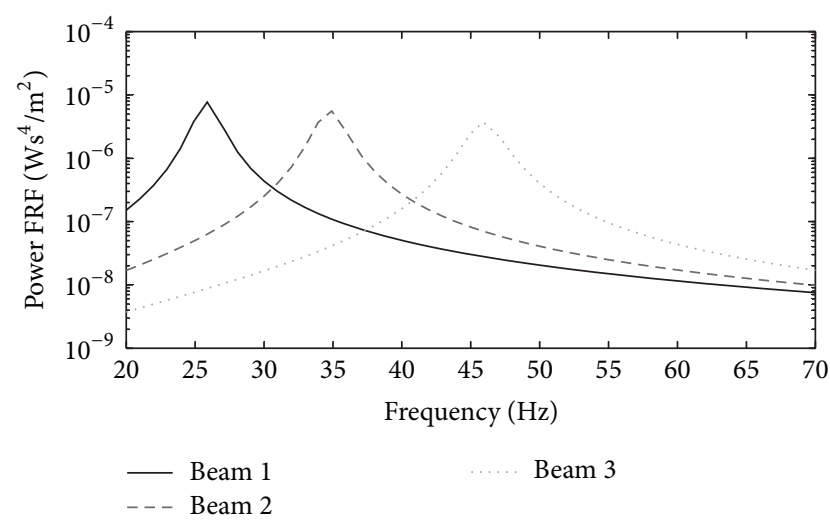

(b) Analytical

FIGURE 13: Power FRF for the three beams with no springs and independent electrical connections to $1 \mathrm{k} \Omega$ resistive loads: (a) experimental measurement, (b) analytical results.

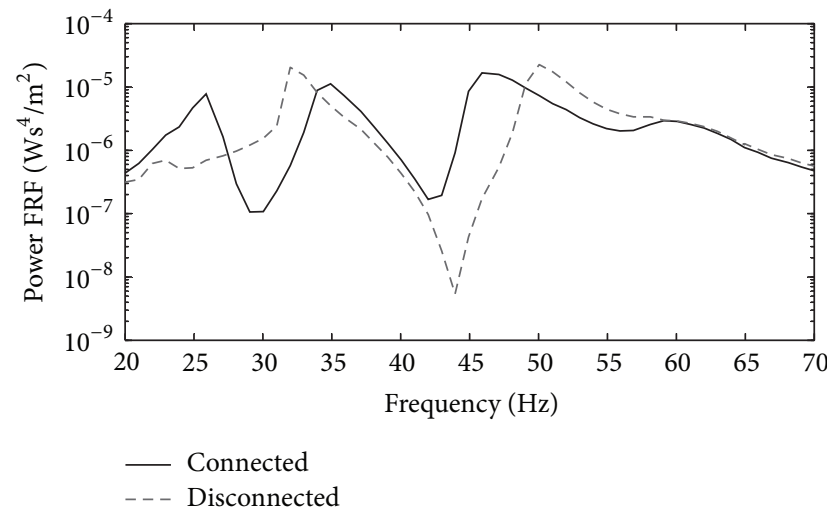

(a) Experimental

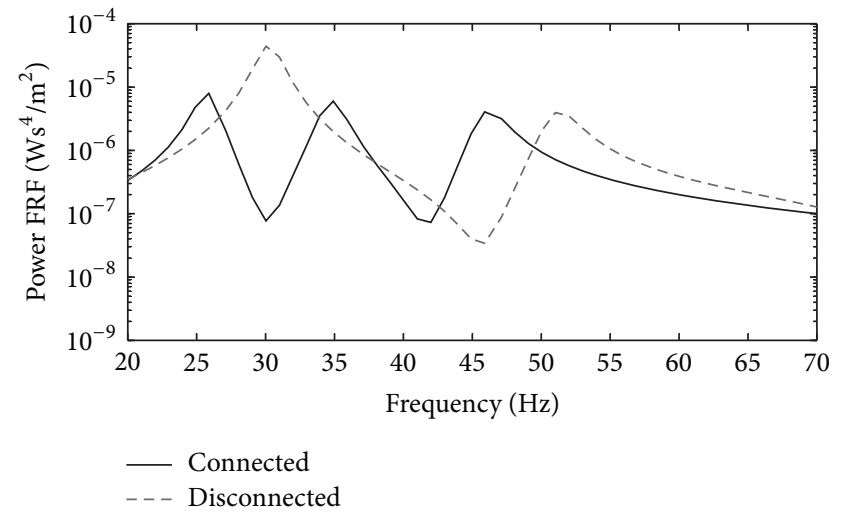

(b) Analytical

FIGURE 14: Power FRF for the three beams with and without springs and electrical connection in parallel to a resistive load of $1 \mathrm{k} \Omega$ :

(a) experimental measurement, (b) analytical results.

\section{Conclusions}

This paper investigated the use of an array of bimorph beams connected by springs as a broadband vibration-based energy harvester. The equations for the voltage and power output of the full system have been deduced based on the analytical solution of the cantilevered piezoelectric energy harvester with Euler-Bernoulli beam assumptions. To study the advantages and disadvantages of the proposed system, the results are compared with those of an array of disconnected beams (with no springs). The analytical model is validated with experimental measurements of three bimorph beams with and without springs.

Both analytical and experimental results show that connecting the array of beams with springs allows increasing the frequency band of operation and increasing the amount of power generated. Thus, the maximal frequency band for a certain array of beams can be extended by connecting them by springs, and at the same time the output power is increased. The performance of this system can be optimized for specific applications by a proper selection of the tip masses and spring constants.
The preliminary experimental results are consistent with the analytical ones, but further research is necessary to improve the experimental setup and to investigate different experimental conditions, such as variations of the springs stiffness.

\section{Conflict of Interests}

The authors declare that there is no conflict of interests regarding the publication of this paper.

\section{References}

[1] S. R. Anton and H. A. Sodano, "A review of power harvesting using piezoelectric materials (2003-2006)," Smart Materials and Structures, vol. 16, no. 3, article R1, 2007.

[2] S. Chalasani and J. M. Conrad, "A survey of energy harvesting sources for embedded systems," in Proceedings of the IEEE SoutheastCon, pp. 442-447, April 2008.

[3] K. A. Cook-Chennault, N. Thambi, and A. M. Sastry, "Powering MEMS portable devices-a review of non-regenerative and regenerative power supply systems with special emphasis on 
piezoelectric energy harvesting systems," Smart Materials and Structures, vol. 17, no. 4, Article ID 043001, 2008.

[4] A. Dewan, S. U. Ay, M. N. Karim, and H. Beyenal, "Alternative power sources for remote sensors: a review," Journal of Power Sources, vol. 245, pp. 129-143, 2014.

[5] A. Harb, "Energy harvesting: state-of-the-art," Renewable Energy, vol. 36, no. 10, pp. 2641-2654, 2011.

[6] S. Saadon and O. Sidek, "A review of vibration-based MEMS piezoelectric energy harvesters," Energy Conversion and Management, vol. 52, no. 1, pp. 500-504, 2011.

[7] H. A. Sodano, D. J. Inman, and G. Park, "A review of power harvesting from vibration using piezoelectric materials," Shock and Vibration Digest, vol. 36, no. 3, pp. 197-205, 2004.

[8] R. J. M. Vullers, R. van Schaijk, I. Doms, C. Van Hoof, and R. Mertens, "Micropower energy harvesting," Solid-State Electronics, vol. 53, no. 7, pp. 684-693, 2009.

[9] R. Caliò, U. B. Rongala, D. Camboni et al., "Piezoelectric energy harvesting solutions," Sensors, vol. 14, no. 3, pp. 4755-4790, 2014.

[10] J. Twiefel and H. Westermann, "Survey on broadband techniques for vibration energy harvesting," Journal of Intelligent Material Systems and Structures, vol. 24, no. 11, pp. 1291-1302, 2013.

[11] S. M. Shahruz, "Design of mechanical band-pass filters for energy scavenging," Journal of Sound and Vibration, vol. 292, no. 3-5, pp. 987-998, 2006.

[12] S. M. Shahruz, "Limits of performance of mechanical bandpass filters used in energy scavenging," Journal of Sound and Vibration, vol. 293, no. 1-2, pp. 449-461, 2006.

[13] S. M. Shahruz, "Design of mechanical band-pass filters for energy scavenging: multi-degree-of-freedom models," Journal of Vibration and Control, vol. 14, no. 5, pp. 753-768, 2008.

[14] J.-Q. Liu, H.-B. Fang, Z.-Y. Xu et al., "A MEMS-based piezoelectric power generator array for vibration energy harvesting," Microelectronics Journal, vol. 39, no. 5, pp. 802-806, 2008.

[15] H. C. Lin, P. H. Wu, I. C. Lien, and Y. C. Shu, "Analysis of an array of piezo-electric energy harvesters connected in series," Smart Materials and Structures, vol. 22, no. 9, Article ID 094026, 2013.

[16] S.-J. Jang, E. Rustighi, M. J. Brennan, Y. P. Lee, and H.-J. Jung, "Design of a 2DOF vibrational energy harvesting device," Journal of Intelligent Material Systems and Structures, vol. 22, no. 5, pp. 443-448, 2011.

[17] L. Tang and Y. Yang, "A multiple-degree-of-freedom piezoelectric energy harvesting model," Journal of Intelligent Material Systems and Structures, vol. 23, no. 14, pp. 1631-1647, 2012.

[18] Z. Yang and J. Yang, "Connected vibrating piezoelectric bimorph beams as a wide-band piezoelectric power harvester," Journal of Intelligent Material Systems and Structures, vol. 20, no. 5, pp. 569-574, 2009.

[19] W. Zhou, G. R. Penamalli, and L. Zuo, "An efficient vibration energy harvester with a multi-mode dynamic magnifier," Smart Materials and Structures, vol. 21, no. 1, Article ID 015014, 2012.

[20] D. S. Nguyen, E. Halvorsen, G. U. Jensen, and A. Vogl, "Fabrication and characterization of a wideband MEMS energy harvester utilizing nonlinear springs," Journal of Micromechanics and Microengineering, vol. 20, no. 12, Article ID 125009, 2010.

[21] S. D. Nguyen and E. Halvorsen, "Nonlinear springs for bandwidth-tolerant vibration energy harvesting," Journal of Microelectromechanical Systems, vol. 20, no. 6, pp. 1225-1227, 2011.

[22] S. Boisseau, G. Despesse, and B. A. Seddik, "Nonlinear h-shaped springs to improve efficiency of vibration energy harvesters,"
Journal of Applied Mechanics, vol. 80, no. 6, Article ID 061013, 2013.

[23] S. Priya, "Advances in energy harvesting using low profile piezoelectric transducers," Journal of Electroceramics, vol. 19, no. 1, pp. 165-182, 2007.

[24] S. Roundy, P. K. Wright, and J. Rabaey, "A study of low level vibrations as a power source for wireless sensor nodes," Computer Communications, vol. 26, no. 11, pp. 1131-1144, 2003.

[25] S. Roundy and P. K. Wright, "A piezoelectric vibration based generator for wireless electronics," Smart Materials and Structures, vol. 13, no. 5, pp. 1131-1142, 2004.

[26] H. A. Sodano, G. Park, and D. J. Inman, "Estimation of electric charge output for piezoelectric energy harvesting," Strain, vol. 40, no. 2, pp. 49-58, 2004.

[27] N. E. Dutoit, B. L. Wardle, and S.-G. Kim, "Design considerations for mems-scale piezoelectric mechanical vibration energy harvesters," Integrated Ferroelectrics, vol. 71, no. 1, pp. 121-160, 2005.

[28] S.-N. Chen, G.-J. Wang, and M.-C. Chien, "Analytical modeling of piezoelectric vibration-induced micro power generator," Mechatronics, vol. 16, no. 7, pp. 379-387, 2006.

[29] A. Erturk and D. J. Inman, "Issues in mathematical modeling of piezoelectric energy harvesters," Smart Materials and Structures, vol. 17, no. 6, Article ID 065016, 2008.

[30] F. Lu, H. P. Lee, and S. P. Lim, "Modeling and analysis of micro piezoelectric power generators for micro-electromechanicalsystems applications," Smart Materials and Structures, vol. 13, no. 1, pp. 57-63, 2004.

[31] S. Jiang, X. Li, S. Guo, Y. Hu, J. Yang, and Q. Jiang, "Performance of a piezoelectric bimorph for scavenging vibration energy," Smart Materials and Structures, vol. 14, no. 4, pp. 769-774, 2005.

[32] A. Erturk and D. J. Inman, "A distributed parameter electromechanical model for cantilevered piezoelectric energy harvesters," Journal of Vibration and Acoustics, vol. 130, no. 4, Article ID 041002, 2008.

[33] A. Erturk and D. J. Inman, "An experimentally validated bimorph cantilever model for piezoelectric energy harvesting from base excitations," Smart Materials and Structures, vol. 18, no. 2, Article ID 025009, 2009. 


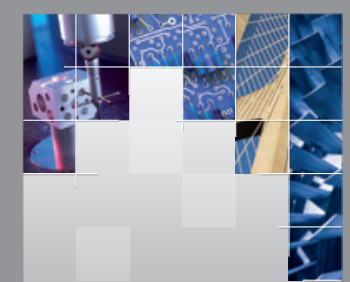

\section{Enfincering}
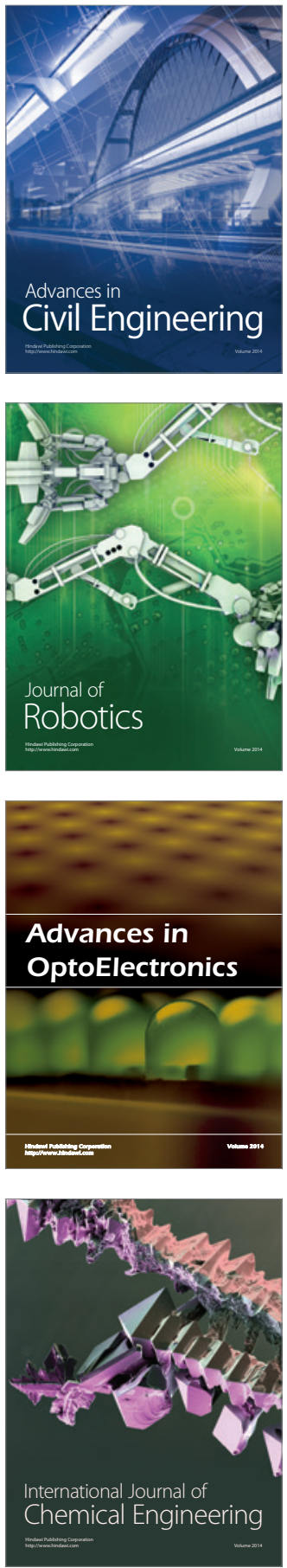

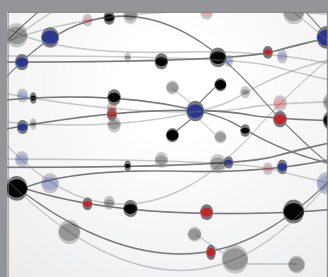

The Scientific World Journal

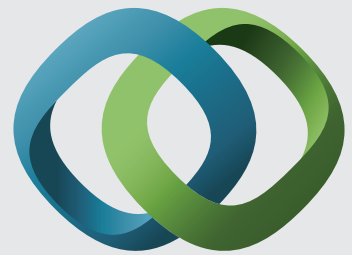

\section{Hindawi}

Submit your manuscripts at

http://www.hindawi.com
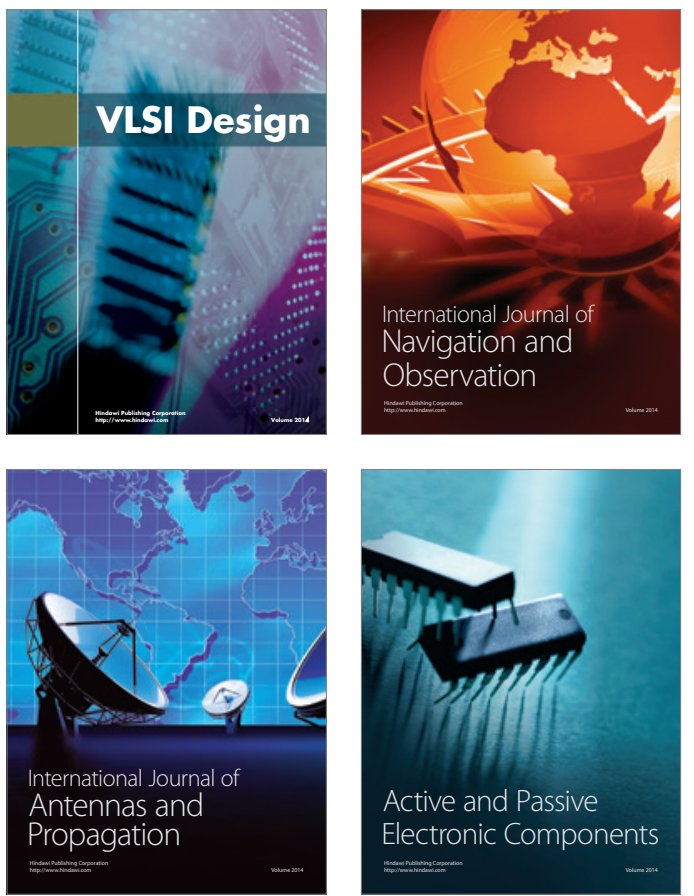
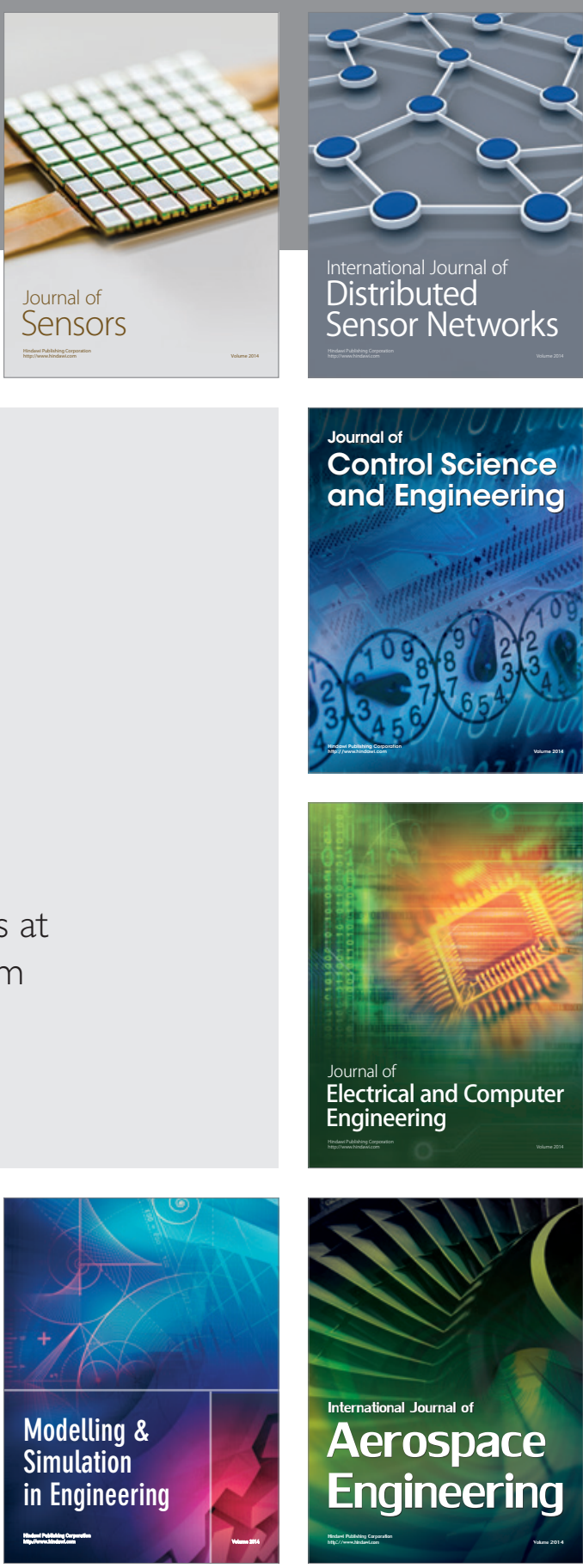

International Journal of

Distributed

Sensor Networks

Journal of

Control Science

and Engineering
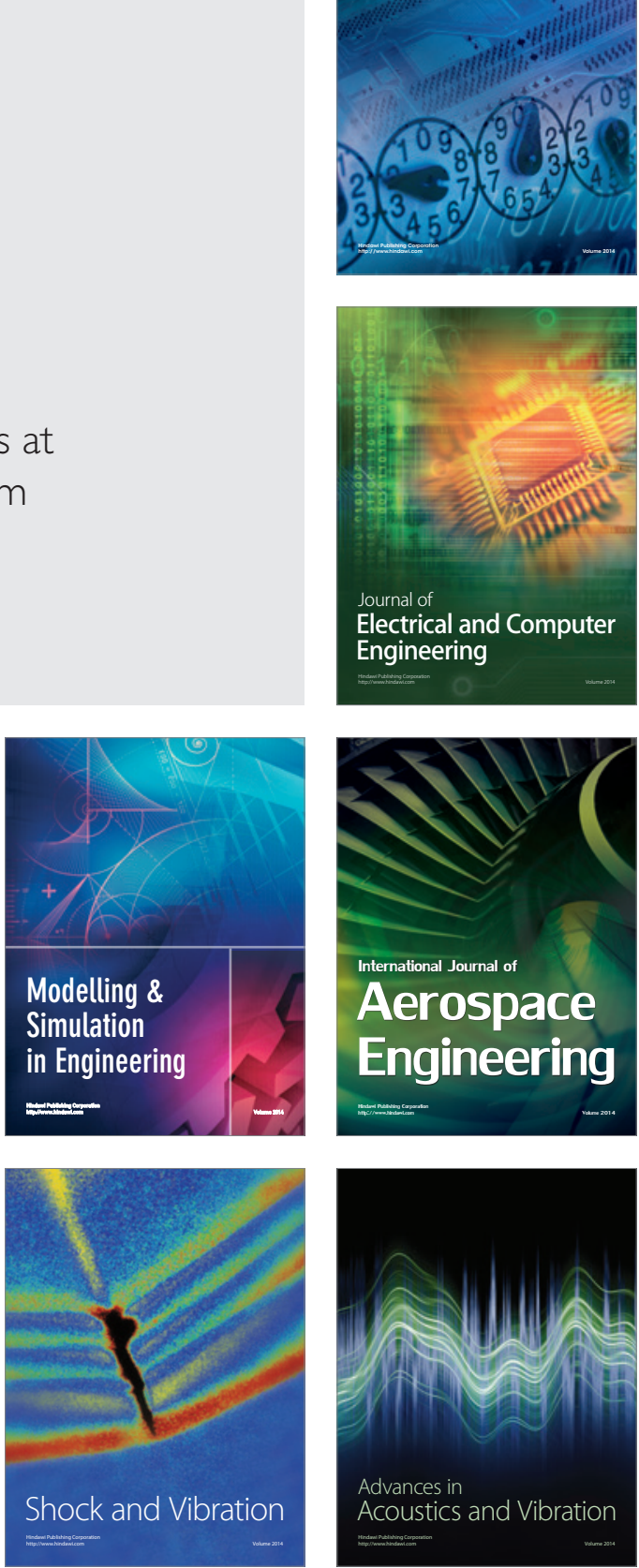